\title{
The Rainfall Intensity Effects on 1-13 GHz UWB-Based 5G System for Outdoor Applications
}

\author{
Joko Suryana \\ School of Electrical Engineering and Informatics, Institut Teknologi Bandung, Bandung, Indonesia \\ Correspondence should be addressed to Joko Suryana; joko.suryana@stei.itb.ac.id
}

Received 6 March 2017; Revised 29 May 2017; Accepted 1 August 2017; Published 20 September 2017

Academic Editor: Ernestina Cianca

Copyright (C) 2017 Joko Suryana. This is an open access article distributed under the Creative Commons Attribution License, which permits unrestricted use, distribution, and reproduction in any medium, provided the original work is properly cited.

\begin{abstract}
This paper reports a research contribution on tropical outdoor channel characterization in 1-13 GHz band for 5G systems. This $1-13 \mathrm{GHz}$ ultra-wideband (UWB) channel characterization is formulated with rain intensity as the most important variable, from $20 \mathrm{~mm} / \mathrm{h}$ to $200 \mathrm{~mm} / \mathrm{h}$. Tropical rain will cause pulse broadening and distorts the transmitted symbols, so the probability of symbol errors will increase. In this research, the bit error rate (BER) performance evaluation is done using both matched filtering or correlator-based receivers. At no rain conditions, BER $10^{-6}$ will be attained at signal to noise ratio (SNR) $5 \mathrm{~dB}$, but at rainfall intensity $200 \mathrm{~mm} / \mathrm{h}$, the BER will fall to $10^{-2}$ for matched filter and $5 \times 10^{-2}$ for correlator-based receivers. For improving the BER performance, an adaptive nonlinear phase equalizer is proposed which adopts multiple allpass biquad infinite impulse response (IIR) filters combined with low-order finite impulse response (FIR) filter to mitigate the nonlinearity phase and differential attenuation of magnitude responses due to antenna and tropical outdoor UWB channel effects. Our simulation results show that the proposed equalizer has worked successfully with BER $10^{-6}$ on the rain rate that is exceeded for $0.01 \%$ of the time $\left(R_{0.01}\right)$ rain intensity or $99.99 \%$ availability. In addition, at rainfall rate $120 \mathrm{~mm} / \mathrm{h}$, the proposed nonlinear phase equalizer can give $9 \mathrm{~dB}$ signal improvement.
\end{abstract}

\section{Introduction}

Like other mobile communication systems, the applications of UWB-based 5G mobile systems, at a specific environment, requires thorough knowledge of the propagation characteristics in that environment. Until now, study of UWB propagation for $5 \mathrm{G}$ applications at outdoor environment is still limited. Many researchers are doing research on outdoor UWB channel characterizations at multipath effects and its path loss only [1-4]. In addition, there is a lack of adequate research $[3,5-7]$ on the effects of atmospheric layers at $1-13 \mathrm{GHz}$, especially in the tropical areas. Therefore, the study of tropical outdoor UWB channel characterization is very relevant to do for preparing UWB-based 5G applications in the near future.

The 1-13 GHz band itself is comprised of two 5G candidate spectrum category: $1-6 \mathrm{GHz}$ as "below $6 \mathrm{GHz}$ spectrum" and $6-13$ as "above $6 \mathrm{GHz}$ spectrum." As $5 \mathrm{G}$ systems develop over time, the $1-6 \mathrm{GHz}$ mobile spectrum bands will be valuable to allow the smooth migration from 4G LTE usage to $5 \mathrm{G}$, while $6-13 \mathrm{GHz}$ spectrum band is attractive in which the existing technology and architecture might be adapted to work in this range, which is closest to existing cellular frequencies. Therefore, this $1-13 \mathrm{GHz}$ spectrum band is of specific interest as it might be able to employ existing cellular technologies with little additional development required. Moreover, the $1-13 \mathrm{GHz}$ spectrum band is also covering the 3.1-10.6 GHz UWB channel which has been adopted for UWB outdoor communication applications at tropical areas.

Unfortunately, the influence of the tropical outdoor channel on the UWB-based 5G communication system performance has not quantitatively assessed in a comprehensive manner yet. In this case, the impulse response of end-to-end tropical outdoor channel will distort the pulse sent by UWB systems. Therefore, it is necessary to formulate the distortion effects of UWB signals by the atmosphere of tropical areas in terms of BER performance of UWB receiver systems.

To maintain the BER performance of UWB systems from outdoor channel distortions and the effects of UWB antenna imperfections, a mitigation technique is required to track 


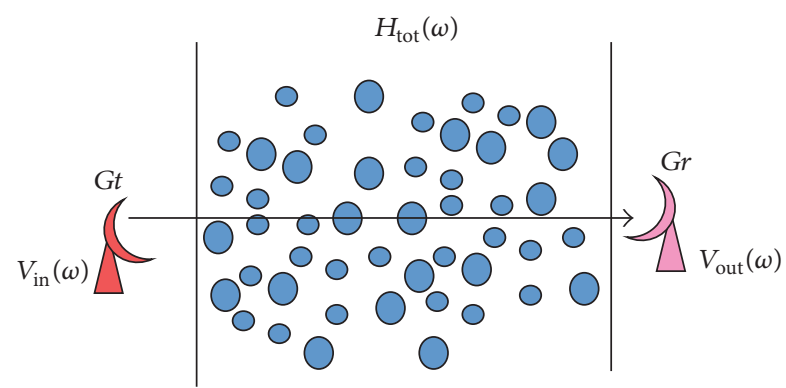

FIgURE 1: Tropical outdoor UWB channel model.

the tropical outdoor channel adaptively. Therefore, there is high demand to develop an adaptive equalization algorithm for compensating the pulse distortion as a result of the phase response nonlinearity as well as the lack uniformity of magnitude response.

The purposes of this research are (1) performing the characterization of tropical outdoor channel $1-13 \mathrm{GHz}$ frequency band with a numerical simulation of UWB signal transmitting through the layer of atmosphere with a variety of rain (the results of numerical simulations are validated with field measurements at the ITB campus environment), (2) calculating the quantification of pulse distortion by atmospheric layer propagation in the tropical areas for the formulation of UWB-based 5G communication systems in terms of BER (the BER performance is formulated with rainfall intensity as the most important parameter on two types of UWB receivers, that is, matched filter-based and the correlator-based receivers), and (3) developing an adaptive nonlinear phase equalization algorithm with low complexity but effectively to overcome the pulse distortion. According to usual performance requirements of $5 \mathrm{G}$ systems, we fixed a target BER equal to $10^{-6}$ and $99.99 \%$ reliability for $500 \mathrm{MBps}$ UWB-based 5G at tropical outdoor applications.

\section{Basic Theory}

2.1. Tropical Outdoor UWB Channel. Tropical outdoor UWB channel is defined as a transmission channel for outdoor UWB applications where, between transmitter (TX) and receiver ( $\mathrm{RX}$ ), there is an atmospheric medium. This atmosphere medium contains the $\mathrm{O}_{2}, \mathrm{H}_{2} \mathrm{O}, \mathrm{CO}_{2}$, and other gases as well as hydrometeors such as rain, clouds, and fog. Figure 1 illustrates the UWB communication system in a tropical outdoor UWB channel. TX transmits an UWB signal towards the RX through atmospheric layer in line of sight manner.

An UWB signal transmission in a tropical atmosphere layer will experience attenuation, phase shift, and the addition of delay time. Transfer function of tropical outdoor UWB channel as Figure 1 can be modeled by the equation frequency region as follows:

$$
H_{\text {tot }}(\omega)=\frac{V_{\text {out }}(\omega)}{V_{\text {in }}(\omega)}=G_{t}(\omega) G_{f}(\omega) G_{a}(\omega) G_{r}(\omega)
$$

with (i) $G_{t}, G_{r}$ are transfer function of Tx or Rx antennas with specific return loss $S_{21}(\omega)$, and, for identical antennas, $G_{t}$ or $G_{r}$ is formulated [8]:

$$
G_{t}(\omega)=G_{r}(\omega)=\sqrt{\frac{2 \pi r c}{j \omega} S_{21}(\omega) e^{j \omega r^{\prime} / c}}
$$

(ii) $G_{f}$ is transfer function of free space propagation as Friis formula in frequency domain [9]:

$$
G_{f}(\omega, d)=\frac{c}{2{ }_{\omega} d} e^{-j \omega d / c}
$$

(iii) $G_{a}$ is transfer function of atmospheric layer

$$
G_{a}(\omega)=\frac{E(d)}{E_{o}}=\exp [-\{\alpha(\omega)+j \beta(\omega)\} d]
$$

with $\alpha$ and $\beta$ defined as [10]

$$
\alpha(\omega)+j \beta(\omega)=\frac{\lambda^{2}}{2 \pi} \int_{0}^{\infty} S_{0}\left[n_{c}, \frac{a}{\lambda}\right] \cdot N(a) \cdot d a .
$$

The atmosphere medium consists of gases such as $\mathrm{O}_{2}$, $\mathrm{H}_{2} \mathrm{O}, \mathrm{CO}_{2}$, and other gases as well as hydrometeors such as rain, clouds, and fog. In this study, the rain-filled medium is modeled by raindrops Mie scattering which are statistically distributed in size as Marshall Palmer distribution [11] with complex permittivity based on Liebe and Hufford measurements [12]. After the impulse response of atmospheric layer is numerically obtained, the next step is to calculate a pulse shape distortion in terms of amplitude, pulse width, and delay time as a function of rainfall intensity: $0,20,50,100$, 150 , and $200 \mathrm{~mm} / \mathrm{h}$. Numerical simulation results are then validated by field measurements using a rain simulator and vector network analyzer $0-13.5 \mathrm{GHz}$ at campus environment.

\subsection{Quantification of the Tropical Atmosphere Effect on UWB} Performance. UWB system BER formulation is limited to UWB system with antipodal modulation and using matched filter and correlator-based receivers as [13]. There are two scenarios formulated BER performance: (1) assuming the antenna system is ideal and (2) assuming the antenna system is realistic.

2.2.1. Performance of Matched Filter-Based Receiver with Ideal UWB Antenna. The energy alteration per bit due to pulse distortions is to be accommodated in the BER equation, as well as the effect of the gain/loss due to the use of the matched filter receiver. Pulse distortion impacts on reducing the energy per bit of signal. Figure 2 illustrates the model of matched filter-based UWB receiver.

$$
\begin{aligned}
H_{m}(\omega) & =\frac{\sqrt{2 \mathrm{BW}}}{(1 / 2 \pi) \int_{-\infty}^{\infty}\left|V_{\text {out }}(\omega)\right|^{2} d \omega} \cdot V_{\text {out }}^{*}(\omega) . \\
V_{m}(\omega) & =H_{m}(\omega) \cdot V_{\text {out }}(\omega) \\
\mathrm{BER}_{m} & =Q\left[\sqrt{\frac{2 \cdot C_{m} \cdot G_{m} \mathrm{BW} \cdot S}{B_{r} N}}\right]
\end{aligned}
$$




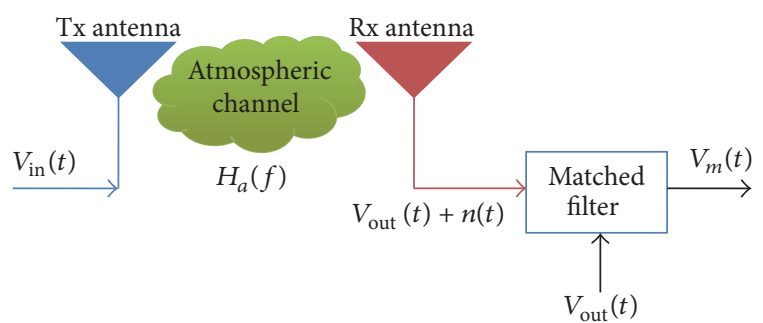

FIGURE 2: Model of matched filter-based UWB receiver.

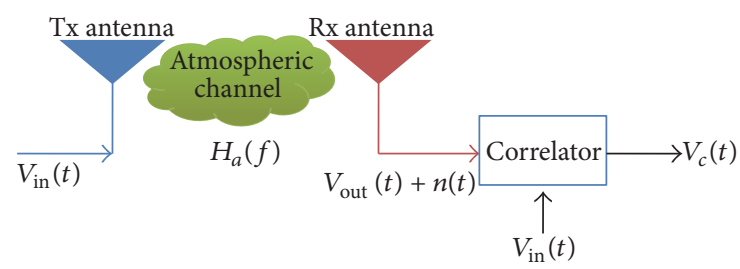

FIGURE 3: Model of correlator-based UWB receiver.

For antipodal modulation, the BER performance of matched filter-based UWB receiver is as (4). In this case, $B_{r}$ is the data rate, BW is the bandwidth, and $C_{m}$ and $G_{m}$, respectively, are the correlation coefficients and the gain of matched filter:

$$
\begin{aligned}
C_{m} & =\frac{\max \left|\int_{-\infty}^{\infty} V_{m}(\omega) e^{j \omega t} d \omega\right|}{\sqrt{\int_{-\infty}^{\infty}\left|V_{\text {out }}(\omega)\right|^{2} d \omega \cdot \int_{-\infty}^{\infty}\left|H_{m}(\omega)\right|^{2} d \omega}} \\
G_{m} & =\frac{\int_{-\infty}^{\infty}\left|V_{m}(\omega)\right|^{2} d \omega}{\int_{-\infty}^{\infty}\left|V_{\text {out }}(\omega)\right|^{2} d \omega} .
\end{aligned}
$$

2.2.2. Performance of Correlator-Based Receiver with Ideal UWB Antenna. As the matched filter-based receiver, the change of energy per bit due to pulse shape distortion also occurs in correlator-based receiver. Figure 3 illustrates the model of correlator-based UWB receiver.

$$
\begin{aligned}
H_{c}(\omega) & =\frac{\sqrt{2 \mathrm{BW}}}{\int_{-\infty}^{\infty}\left|V_{\mathrm{in}}(\omega)\right|^{2} d \omega} \cdot V_{\mathrm{in}}^{*}(\omega) \\
V_{c}(\omega) & =H_{c}(\omega) \cdot V_{\text {out }}(\omega) \\
\mathrm{BER}_{c} & =Q\left[\sqrt{\frac{2 \cdot C_{c} \cdot G_{c} \cdot \mathrm{BW} \cdot S}{B_{r} N}}\right] .
\end{aligned}
$$

For antipodal modulation, the BER performance of correlator-based UWB receiver is as (8) with $B_{r}$ being the data rate, BW the bandwidth, and $C_{c}$ and $G_{c}$, respectively, the correlation coefficients and the gain of correlator-based receiver:

$$
\begin{aligned}
C_{c} & =\frac{\max \left|\int_{-\infty}^{\infty} V_{c}(\omega) e^{j \omega t} d \omega\right|}{\sqrt{\int_{-\infty}^{\infty}\left|V_{\text {out }}(\omega)\right|^{2} d \omega \cdot \int_{-\infty}^{\infty}\left|H_{c}(\omega)\right|^{2} d \omega}} \\
G_{c} & =\frac{\int_{-\infty}^{\infty}\left|V_{c}(\omega)\right|^{2} d \omega}{\int_{-\infty}^{\infty}\left|V_{\text {out }}(\omega)\right|^{2} d \omega} .
\end{aligned}
$$

\subsubsection{Performance of UWB Receivers with Realistic UWB} Antenna. In the previous sections, we have discussed the performance evaluation of UWB communications systems in dispersive outdoor tropical UWB channels with ideal antenna. This section presents a derivation of BER equation for tropical outdoor channel with realistic antennas. The influence of distortion by the antenna system is very dependent on the parameters $S_{11}$ and $S_{21}$ and the gain of the antenna system. The effects of $S_{11}$ and $S_{21}$ parameters and antenna gain can be represented by a fidelity value which is defined in [14].

$$
\begin{aligned}
F( & r(t), p(t)) \\
& =\max _{\tau}\left|\int_{-\infty}^{\infty} \frac{r(t) \cdot p(t-\tau)}{\sqrt{\int_{-\infty}^{\infty}|r(t)|^{2} d t \int_{-\infty}^{\infty}|p(t)|^{2} d t}} d t\right|,
\end{aligned}
$$

where fidelity profile $F(r(t), p(t))$ is a measure of similarity between $r(t)$ and $p(t)$. In this case $r(t)$ is a received signal after passing through the antennas (both transmit and receive sides) and $p(t)$ is a template signal at receiver. When an UWB system uses realistic antenna with specific return loss $S_{11}$, the gain, the transfer function $S_{21}$, and the BER equation for both receiving systems become

$$
\begin{aligned}
& \mathrm{BER}_{m}=Q\left(\sqrt{\frac{2 F C_{m} G_{m} \mathrm{BW} \cdot S}{B_{r} \cdot N}}\right) \\
& \mathrm{BER}_{c}=Q\left(\sqrt{\frac{2 F C_{c} G_{c} \mathrm{BW} \cdot S}{B_{r} \cdot N}}\right) .
\end{aligned}
$$

It is assumed that we use same tropical outdoor channel model as in previous section, but in this case, we used TX and $\mathrm{RX}$ realistic antennas with specific fidelity profile, $F$.

2.3. Adaptive Nonlinear Phase Equalizer. In this study, it is proposed an adaptive nonlinear phase equalizer for compensating the pulse distortion by combining allpass biquad IIR [15] and FIR filters with a channel estimator block as Figure 4.

Allpass biquad IIR filter is used to compensate nonlinear phase response that comes from the tropical outdoor channel and the antenna. Meanwhile, to compensate the magnitude response, we use low-order FIR filter cascaded with the allpass biquad IIR filter. For measuring the instantaneous channel condition, channel estimator with its training pattern signal is used to calculate the channel transfer function periodically.

In this case, the end-to-end transmission channel is represented by convolving the tropical outdoor UWB channel 


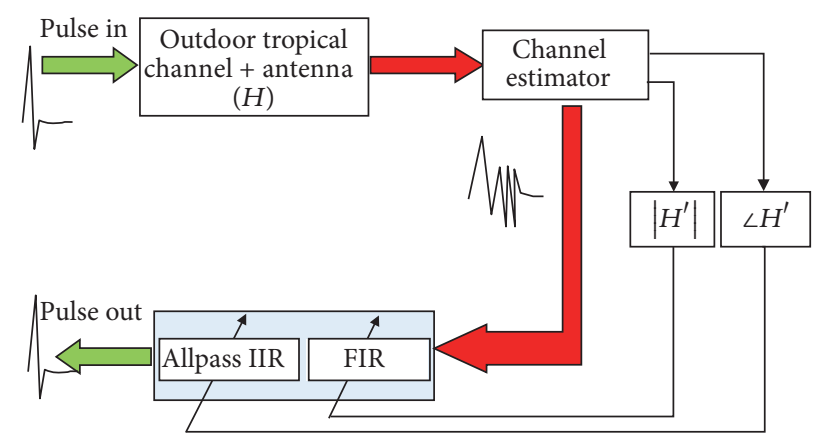

FIGURE 4: Block diagram of proposed adaptive nonlinear phase equalizer.

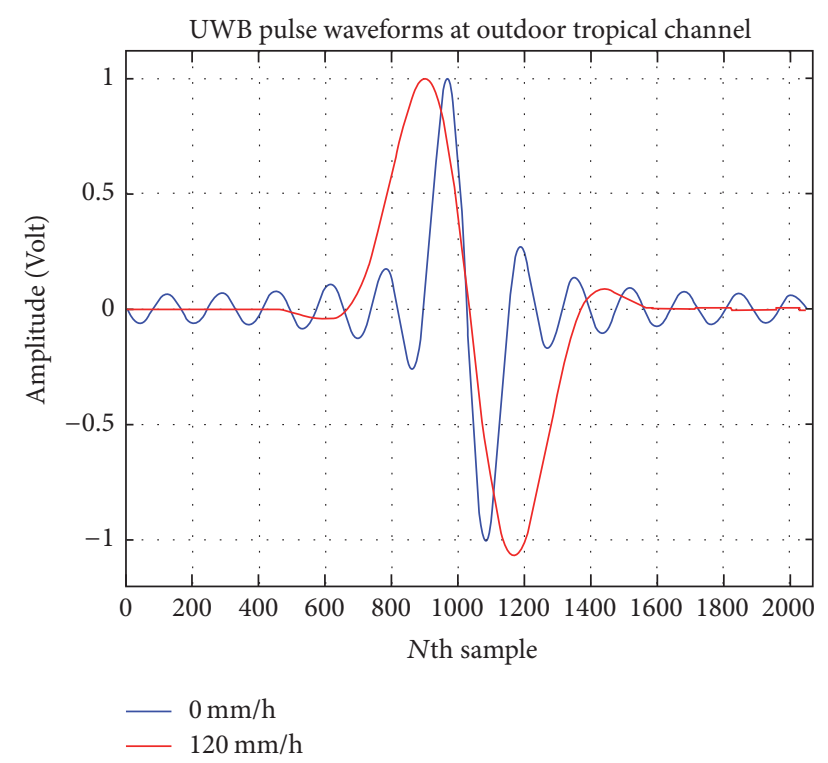

FIGURE 5: Pulse shapes in a tropical outdoor UWB channel.

with a dispersive UWB antenna system. The rainfall rate used in this simulation is $R_{0.01}$ of Bandung [16]; the antenna selected is Log Periodic antenna which has bandwidth at $2-8 \mathrm{GHz}$ [17]. It is assumed that the distance between TX and RX is 10 -meter long. A Gaussian 0.1333 ns pulse shapes after passing through the tropical outdoor UWB channel at $0 \mathrm{~mm} / \mathrm{h}$ and $120 \mathrm{~mm} / \mathrm{h}$ and can be seen in Figure 5.

Antenna used in this simulation is a Log Periodic antenna with wide bandwidth but has a dispersive impulse response. As in [17], Log Periodic is one of antenna classes which have wide bandwidth and dispersive impulse response. Frequency and time domain characteristics of the $2-8 \mathrm{GHz}$ Log Periodic antenna can be seen in Figure 6.

\section{Results and Discussion}

3.1. Characterization of Tropical Outdoor UWB Channel. The numerical simulation results of the attenuation coefficient per $\mathrm{km}$ and phase coefficient per $\mathrm{km}$ [18] can be seen in Figure 7. When the distance between TX and RX is known, then by using the curve in Figure 7, we can obtain the magnitude response and the phase response of the tropical outdoor UWB channel.

A transfer function of a tropical outdoor UWB channel can then be calculated by combining the magnitude and phase responses as a function of frequency and rainfall intensity at $0,20,50,100,150$, and $200 \mathrm{~mm} / \mathrm{h}$. And by using the inverse Fourier transform, we can determine the channel impulse response.

In this research, author used short range of tropical outdoor communication model for both 4-meter and 10meter distance. The reason behind choosing these short range comes from the fact that our rain simulator facility as described on Section 3.2 has maximum length of about 10 meter. Due to its rain attenuation proportionality properties to range, our short range simulation and measurement results will lead us to estimate the rain effects for longer range as $5 \mathrm{G}$ realistic applications.

3.1.1. Numerical Simulation Results of Impulse Response of Tropical Outdoor UWB Channel at $d=4$ Meter. Figure 8 is numerical simulation results of the channel impulse response of tropical outdoor at $1-13 \mathrm{GHz}$ band as a function of on frequency on a variety of rainfall intensity for TX and RX distance $d=4$ meters.

From Figure 8, it can be seen that as the rainfall intensity becomes greater, then the impulse response will be more broadened and delayed and its amplitude will be reduced. However, at distance between TX and RX antenna $d=4$ meters, the influence of rainfall intensity is small enough.

3.1.2. Numerical Simulation Results of Impulse Response of Tropical Outdoor UWB Channel at $d=10$ Meters. In our second calculation of impulse response of tropical outdoor UWB channel, we assumed that the distance between the TX and RX antenna is 10 meters. By comparing the results of calculations on two different distances $d$, it is expected to see the effect of distance on the impulse response profiles. Figure 9 shows that, at distance $d=10$ meters, the pulse distortion is higher than at $d=4$ meters.

At very high rainfall intensity $(200 \mathrm{~mm} / \mathrm{h})$, the impulse response has amplitude shrinking, more broadening, and delay.

From the numerical results at a distance of $d=4 \mathrm{~m}$ and $d=10 \mathrm{~m}$ as shown in Figures 8 and 9 we may conclude that the intensity of tropical rainfall affects the changing shape of the channel impulse response distortion which occurred with an increasing delay, amplitude reduction, and pulse duration broadening. In this case, the distance also gives effect proportional to the pulse distortion.

3.2. Tropical Outdoor UWB Channel Measurements. Figure 10 shows the configuration of UWB pulse propagation measurements in an outdoor environment.

This measurement setup consists of an array of water sprayers, vector network analyzer, rain gauge, and TX and RX antennas. The maximum range of our outdoor measurement which can be achieved is 10 meters with a variation of rainfall 

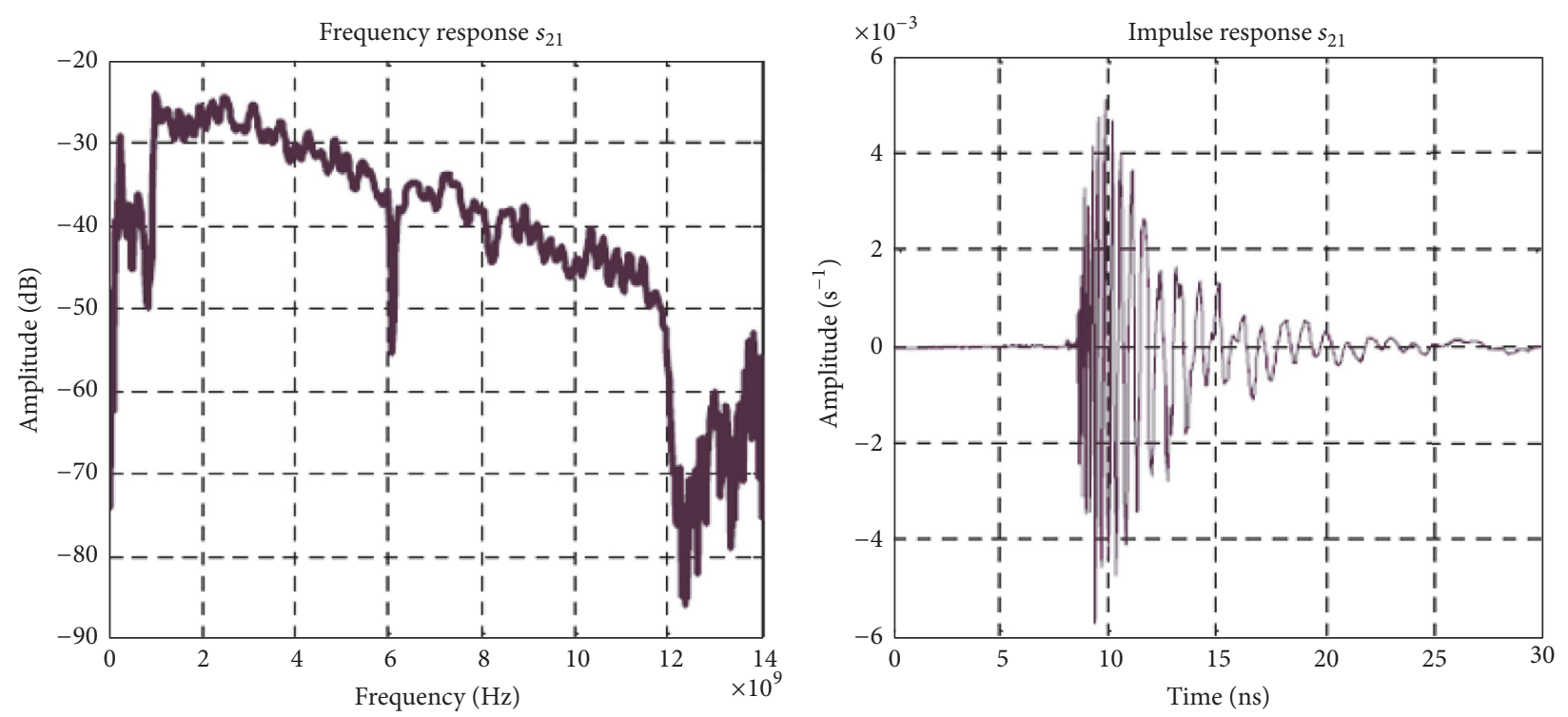

FIGURE 6: Log periodic antenna characteristics.

intensity from 0 to $200 \mathrm{~mm} / \mathrm{h}$. The device used in outdoor UWB channel measurement consists of the following:

(1) Vector Network Analyzer (VNA) 0-13 GHz

(2) Array of water sprayers as a tropical rain simulator that has intensity control to simulate different rainfall intensities: 0, 20, 50, 100, 150, and $200 \mathrm{~mm} / \mathrm{h}$

(3) Rain gauge, a device for measuring rainfall intensity

(4) A pair of 1-13 GHz UWB antennas as the photograph in Figure 11 and their frequency and time domain characteristics as in Figure 12.

3.2.1. Measurement Results of Tropical Outdoor UWB Channel for $d=4$ Meters. Tropical outdoor channel measurements were performed using the frequency domain approach as $S_{21}$ parameter. To display the tropical outdoor channel impulse response in time domain, the measured $S_{21}$ parameter is then processed by inverse Fourier transform using MATLAB software. Figure 13 is the result of the channel impulse response measurements of tropical outdoor band $1-13 \mathrm{GHz}$ at different rainfall intensity for TX and RX distances at $d=4$ meters before and after deconvolution and noise filtering.

Deconvolution process conducted on raw impulse response data is intended to eliminate the distortion effects of TX and RX antennas. To eliminate the noise from the raw data, we used simple filtering with a moving average filter. From Figure 13 we can see that as the rainfall intensity becomes greater, then the impulse response will be broadened and more delayed and its amplitude will reduce. In this case, at $d=4$ meters, the influence of rainfall intensity is not too large, so the impulse response of the tropical outdoor channel almost coincides. for $d=10$ Meters. In our second measurement of tropical outdoor UWB channel, we set the distance between the antenna TX and RX antenna at 10 meters. By comparing the measurement results of two different distances $d$, it is expected to see the effect of distance to the channel impulse response.

Figure 14 shows that influence of rain intensity on the distance $d=10$ meters is higher than the measurement result at $d=4$ meters. The impulse response of tropical outdoor UWB channel at very high rainfall $(200 \mathrm{~mm} / \mathrm{h})$ has amplitude shrinking, so the impulse response width and a shift towards the main axis become larger than when there is no rain $(0 \mathrm{~mm} / \mathrm{h})$.

When we compare the results of numerical simulation and measurement results of the channel impulse responses as Figures 8, 9, 13, and 14, we see that there is a strong correspondence between the measurement results with simulation results both for the pulse broadening, the time shifted, and the amplitude reduction of impulse responses.

The curves in Figures 15, 16, and 17 consecutively quantitatively showed us pulse broadening, the time shifted, and the amplitude reduction of impulse responses at distance $d=4$ and $d=10$ meters as a function of rainfall intensity.

The three curves confirmed that the range and variation of rainfall intensity impact on pulse broadening, the time shifted, and the amplitude reduction of impulse responses.

3.3. Quantification of the Tropical Atmosphere Effect on UWBBased 5G Performance. In this scenario, UWB-based 5G systems is assumed to have $500 \mathrm{MBps}$ with antipodal modulation for outdoor applications at tropical areas. The UWBbased $5 \mathrm{G}$ system operates at $3.1-10.6 \mathrm{GHz}$ for achieving $7.5 \mathrm{GHz}$ with very low output power density. Ideal Antenna. Figure 18 presents the BER performance of 


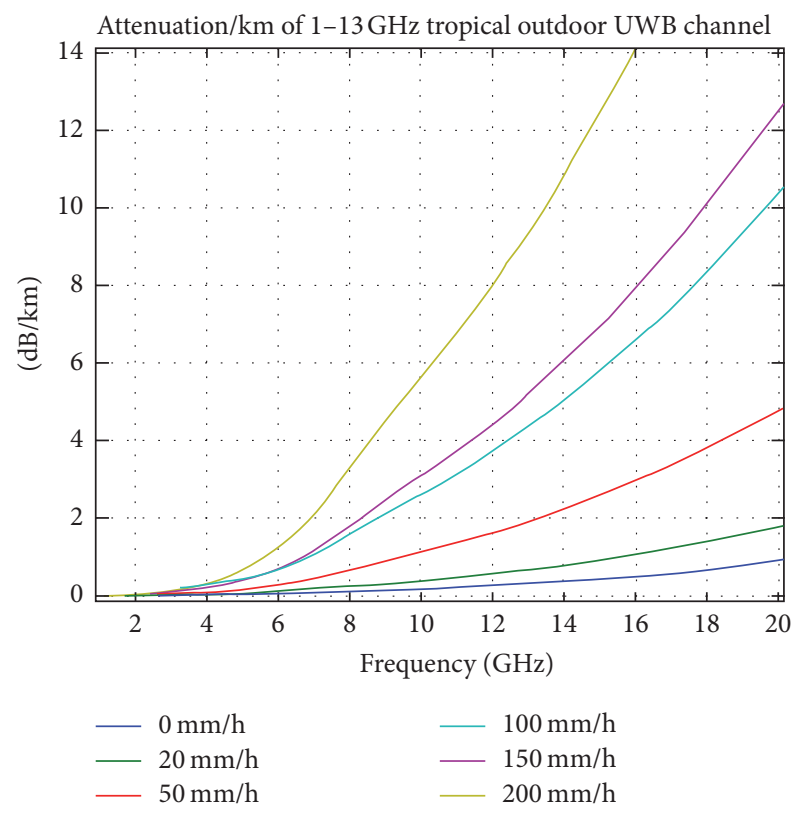

Phase shifted/km of 1-13 GHz tropical

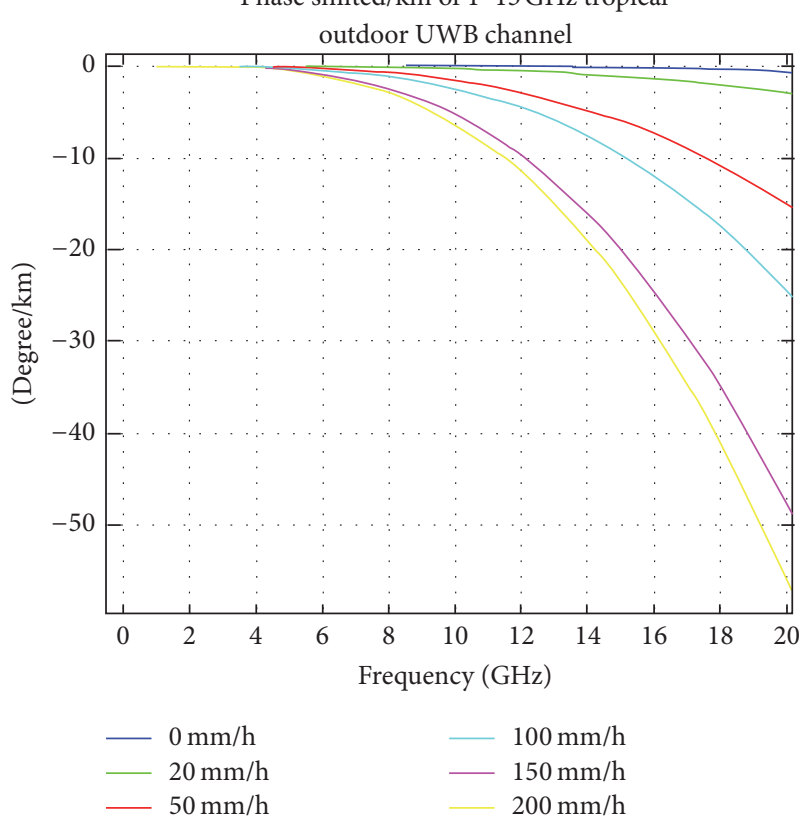

FIGURE 7: Magnitude and phase response of tropical outdoor UWB channel at $1-13 \mathrm{GHz}$ band.

UWB-based 5G system using matched filter-based receiver with ideal antenna at 10 meter distance and bitrate $500 \mathrm{Mbps}$ for several of rainfall intensities. From this figure we can see that the BER performance curve decreases with increasing rainfall intensity which occurs along the path between the TX and RX.

At $10^{-6}$ BER performance, the SNR requirements must be worth $5,8,12,16,17.5$, and $18 \mathrm{~dB}$ for rainfall intensity conditions, respectively, $0,20,50,100,150$, and $200 \mathrm{~mm} / \mathrm{h}$. In other words, for keeping $10^{-6}$ BER performance continuously



FIGURE 8: Simulated impulse response of tropical outdoor UWB channel on $d=4 \mathrm{~m}$.

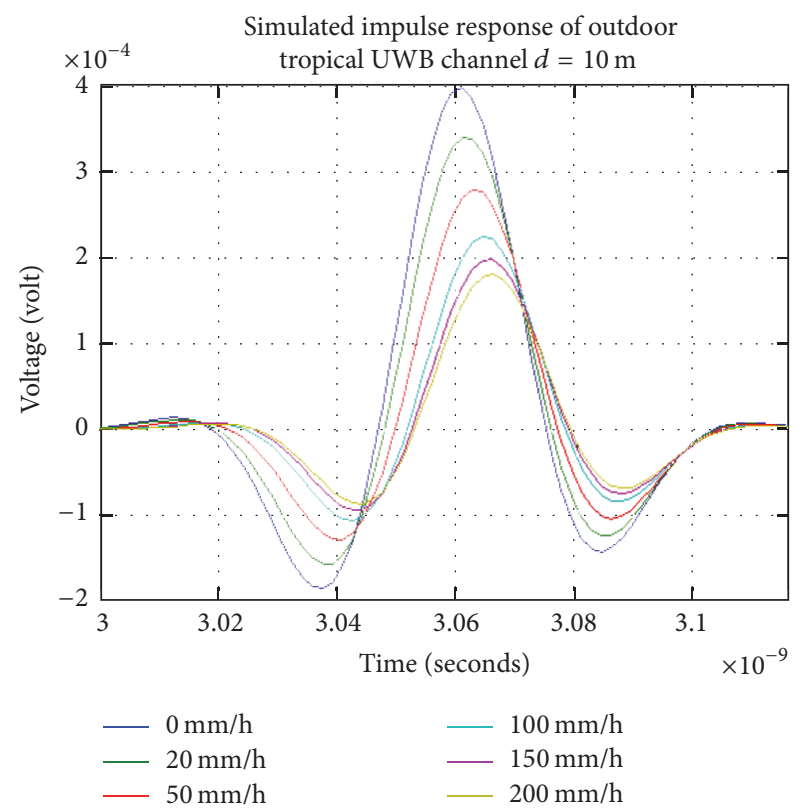

FIGURE 9: Channel impulse response of tropical outdoor UWB on $d=10 \mathrm{~m}$.

in various conditions of rain, the required fading margin is minimum $13 \mathrm{~dB}$.

Meanwhile, if the benchmark performance using $R_{0.01}$ rainfall intensity is $120 \mathrm{~mm} / \mathrm{h}$ for Bandung, the required fading margin is $11 \mathrm{~dB}$. The BER curves in Figure 18 also show that, at fixed SNR conditions, such as $5 \mathrm{~dB}$, then the BER performances are, respectively, $10^{-6}, 10^{-4}, 10^{-3}$, and 


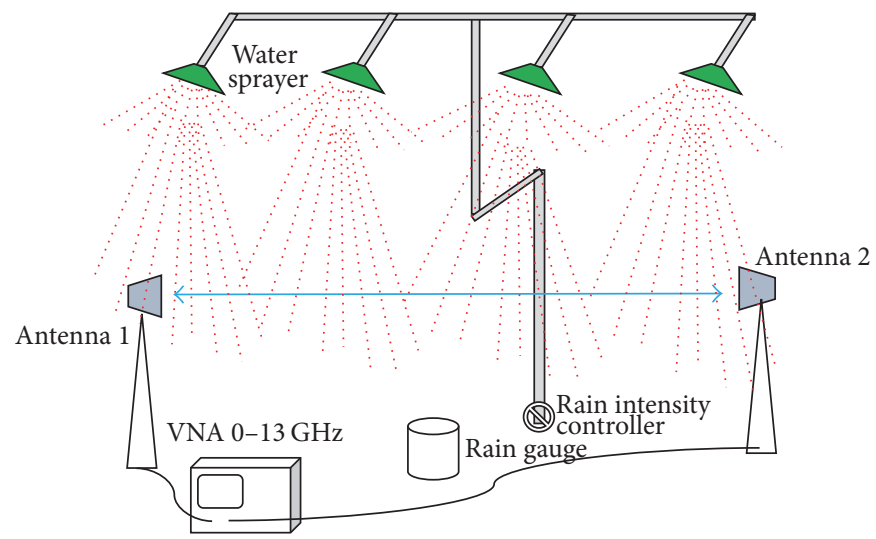

FIGURE 10: Tropical outdoor channel measurement setup.



FIGURE 11: Photograph of antenna used for UWB channel measurements.

$10^{-2}$ for rainfall intensity at $0 \mathrm{~mm} / \mathrm{h}, 20 \mathrm{~mm} / \mathrm{h}, 50 \mathrm{~mm} \mathrm{~h}$, and $100 \mathrm{~mm} / \mathrm{h}$.

3.3.2. BER Performance of Correlator-Based Receiver with Ideal Antenna. Here as in Figure 19, the BER performance of UWB system is using correlator-based receiver with ideal antenna at 10 meters distance and bitrate $500 \mathrm{Mbps}$ for several of rainfall intensities. From this figure we see that BER curves decrease with increasing rainfall intensity which occurs along the path between the TX and RX. At $10^{-6}$ BER performance, SNR requirements should be worth $7,10,14,18,20$, and $22 \mathrm{~dB}$ for rainfall intensity conditions, respectively, 0,20 , $50,100,150$, and $200 \mathrm{~mm} / \mathrm{h}$. In other words, to obtain the BER performance $10^{-6}$ continuously, the necessary due fading margin is minimum $15 \mathrm{~dB}$.

BER curves at Figure 19 also show that, at fixed SNR conditions, such as $7 \mathrm{~dB}$, the BER then, respectively, is $10^{-6}, 10^{-5}$, $10^{-3}$, and $5 \times 10^{-1}$ for rainfall $0 \mathrm{~mm} / \mathrm{h}, 20 \mathrm{~mm} / \mathrm{h}, 50 \mathrm{~mm} / \mathrm{h}$, and $100 \mathrm{~mm} / \mathrm{h}$. Comparing the BER performance curve between matched filter and correlator-based receiver, we can be see that matched filter is $3-5 \mathrm{~dB}$ better than correlator-based receiver.
3.3.3. BER Performance by Tropical Outdoor Channel and Realistic UWB Antenna. The simulation results of BER performance evaluation of tropical outdoor channel with realistic antenna can be seen in Figures 20 and 21 with matched filter and correlator-based receiver, respectively. From the curves in Figure 20, we can see that the UWB system performance is strongly influenced by the transient responses of antenna and tropical outdoor channel. In the matched filter-based receiver, the use of realistic UWB antenna causes a decrease of 8-9 dB SNR, while the correlator-based receiver decreases SNR falls by $9-10 \mathrm{~dB}$.

3.4. UWB-Based 5G Bit Rate Reduction due to Tropical Outdoor UWB Channel and Dispersive Antenna. The effects of rainfall intensity and the dispersive antenna to a reduction in bitrate of UWB communication system is summarized as in Figure 22. The specifications of UWB system are BER at $10^{-6}$ maintained, bandwidth of $7.5 \mathrm{GHz}$, and bitrate in additive white Gaussian noise (AWGN) condition at $500 \mathrm{Mbps}$ and 10 meters distance.

From Figure 22 we also can see that the intensity of rainfall has a direct impact on the reduction in bitrate of UWB communication systems. The higher the rainfall the greater the reduction in data rate for all scenarios. In an ideal scenario with ideal antenna, at rainfall intensity $200 \mathrm{~mm} / \mathrm{h}$, the bitrate went down from $500 \mathrm{Mbps}$ (without rain) to $25 \mathrm{Mbps}$ for the matched filter and $15 \mathrm{Mbps}$ for the correlator-based receiver. Meanwhile, in realistic antenna scenarios, at rainfall intensity $200 \mathrm{~mm} / \mathrm{h}$, the bitrate declined from $50 \mathrm{Mbps}$ (without rain) to $2 \mathrm{Mbps}$ for matched filter and $1 \mathrm{Mbps}$ for the correlatorbased receiver.

3.5. Adaptive Nonlinear Phase Equalizer. The proposed adaptive nonlinear phase equalizer is used for mitigating the distortions due to tropical outdoor channel and comes from dispersive antenna. The target performance criteria of UWBbased 5G system for outdoor applications are using antipodal modulation, operating frequency from 3.1 to $10.6 \mathrm{GHz}$, required SNR set to $10 \mathrm{~dB}$ for $R=500 \mathrm{Mbps}$, power margin provided as $5 \mathrm{~dB}$, minimum BER $10^{-6}$ with availability $99.99 \%$ 

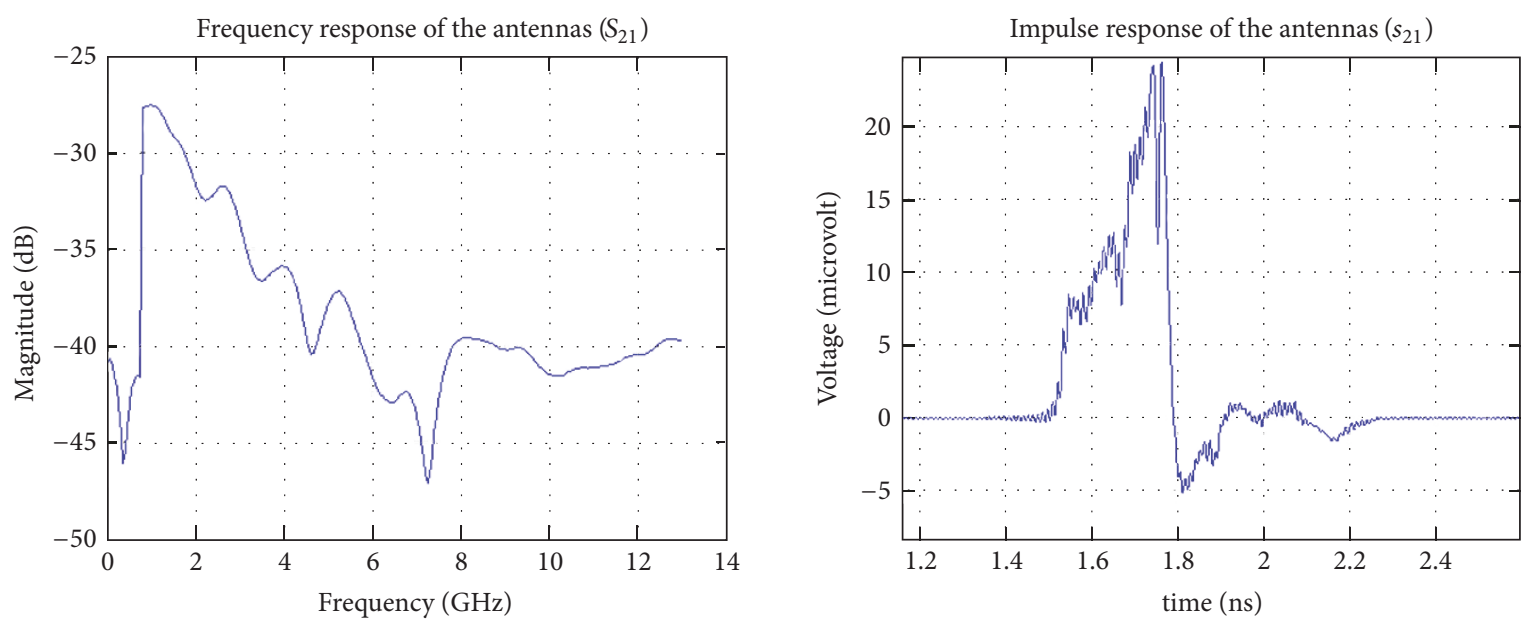

FIGURE 12: Frequency and time domain characteristic of antenna used for UWB channel measurements.

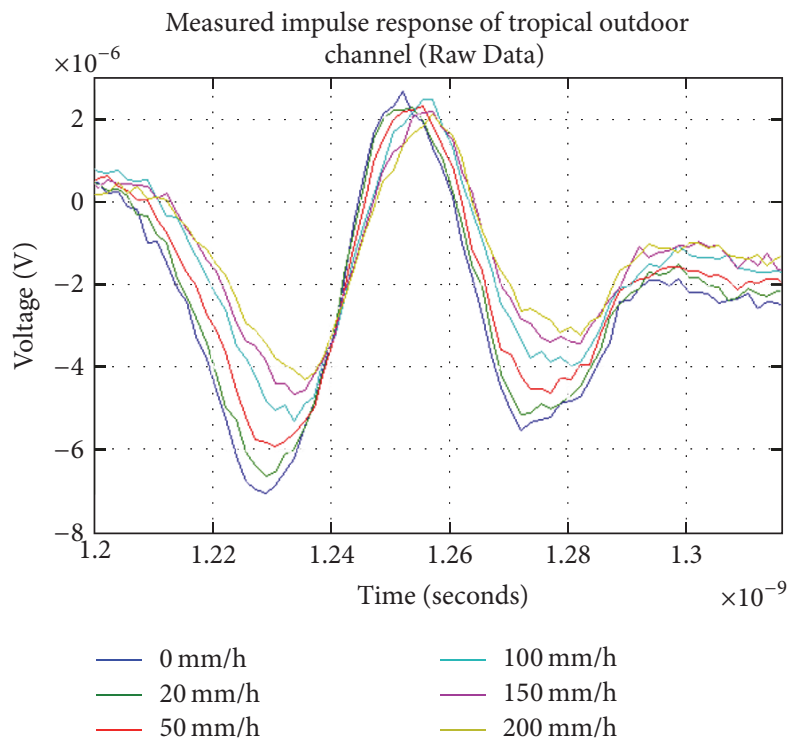

(a)

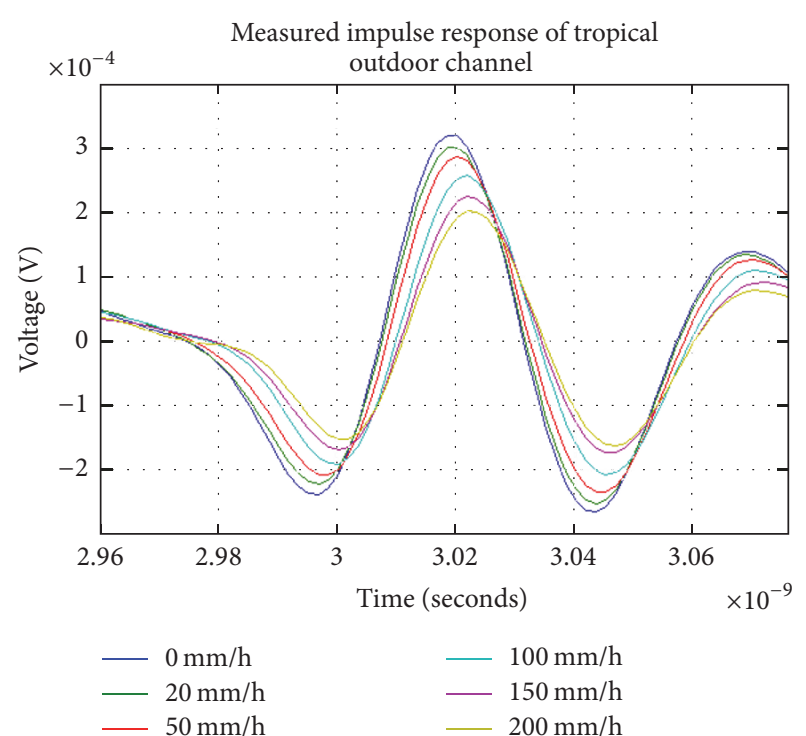

(b)

FIGURE 13: Measured impulse response before (a) and after (b) deconvolution and noise filtering at $d=4$ meter.

for rainfall $R_{0.01}=120 \mathrm{~mm} / \mathrm{h}$, and the receiver based on both matched filter or correlator.

In this scenario, we proposed an adaptive nonlinear phase equalizer based on allpass biquad IIR order 6 cascaded to FIR filter order 6 . The magnitude and phase responses of allpass biquad IIR order 6 and its poles/zeros structure are shown in Figures 23 and 24.

3.5.1. BER Improvement of Matched Filter before and after Nonlinear Compensation. The curve as in Figure 25 shows the BER performance of matched filter-based receiver with and without nonlinear phase compensation. The red portion of the graph states that the BER performances are under our technical requirements $\left(\mathrm{BER}>10^{-6}\right)$.
From this figure, we can see that improvement has occurred around $10 \mathrm{~dB}$ SNR compared to the BER performance of matched filter-based receiver without phase compensation. Thus an UWB system with a minimum SNR $10 \mathrm{~dB}$ and $5 \mathrm{~dB}$ fading margin can still be working well at BER $10^{-6}$.

In this case, phase compensation is done due to the contribution of rainfall intensity $120 \mathrm{~mm} / \mathrm{h}$, but also done on the nonlinearity phase response of antenna. Therefore, the application of phase equalization on matched filter-based receiver can ensure the UWB system works on the availability of $99.99 \%$ for the $R_{0.01}=120 \mathrm{~mm} / \mathrm{h}$ at Bandung.

As for rainfall above $120 \mathrm{~mm} / \mathrm{h}$, BER performance falls below the desired performance requirements. However, 


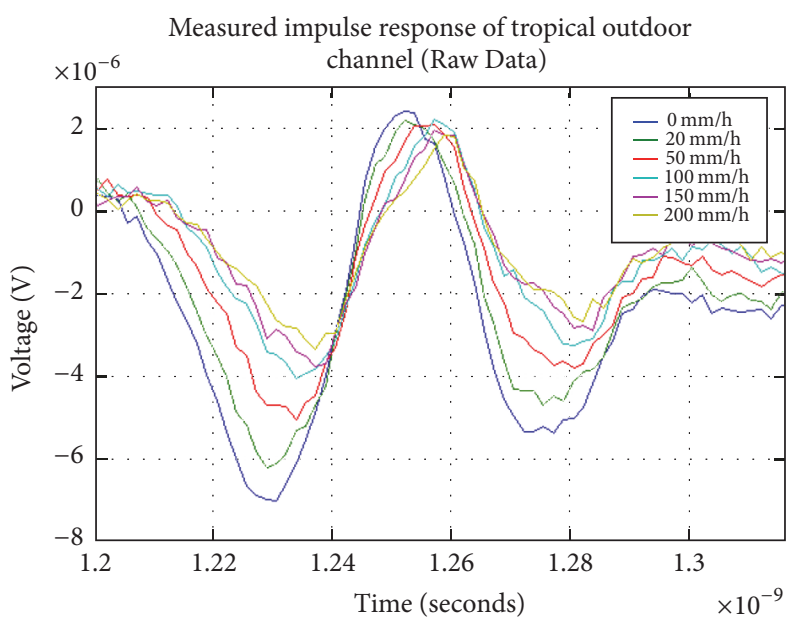

(a)

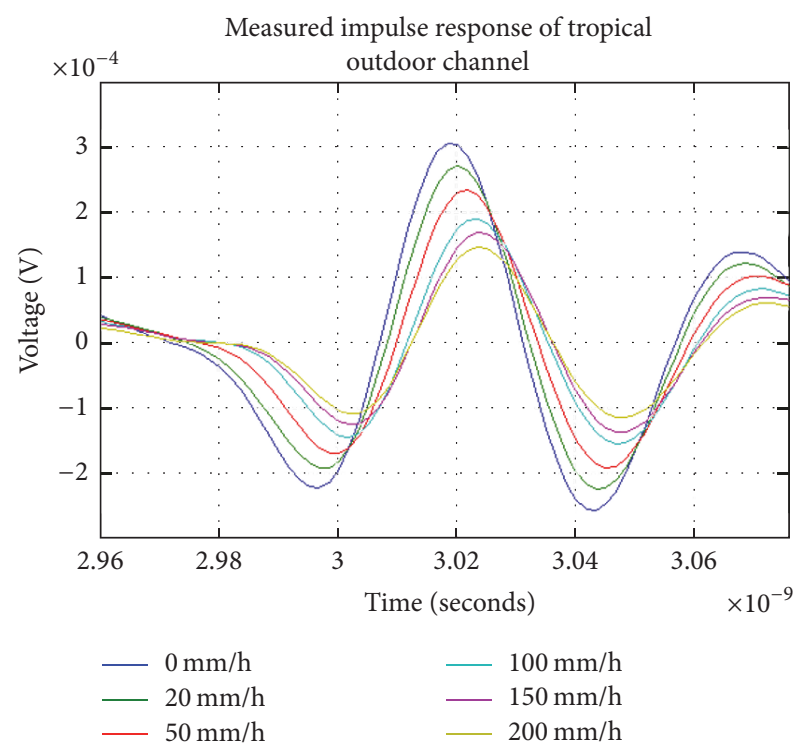

(b)

FIGURE 14: Measured impulse response before (a) and after (b) deconvolution and noise filtering at $d=10$ meters.

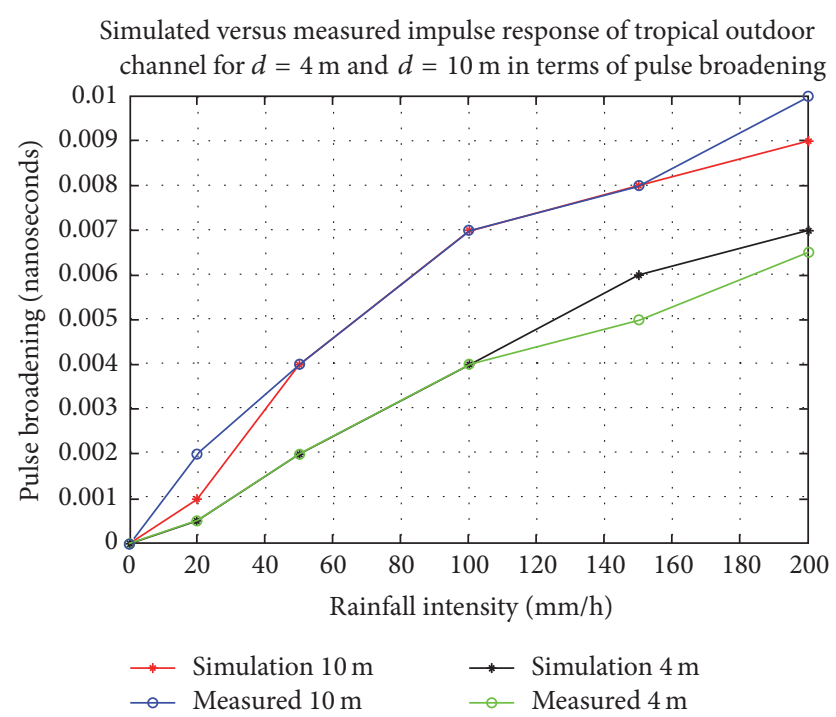

FIGURE 15: Simulated versus measured pulse broadening of tropical outdoor channel at $d=4$ meters and $d=10$ meters.

rainfall intensity above $120 \mathrm{~mm} / \mathrm{h}$ has an opportunity which occurs $<0.01 \%$ a year so that is not statistically significant.

3.5.2. BER Improvement of Correlator before and after Nonlinear Compensation. The simulation of BER performance improvement by nonlinear phase compensator for correlatorbased receiver can be seen in Figure 26.

The BER curve as in Figure 26 shows that improvement has occurred around $10 \mathrm{~dB}$ SNR compared to the

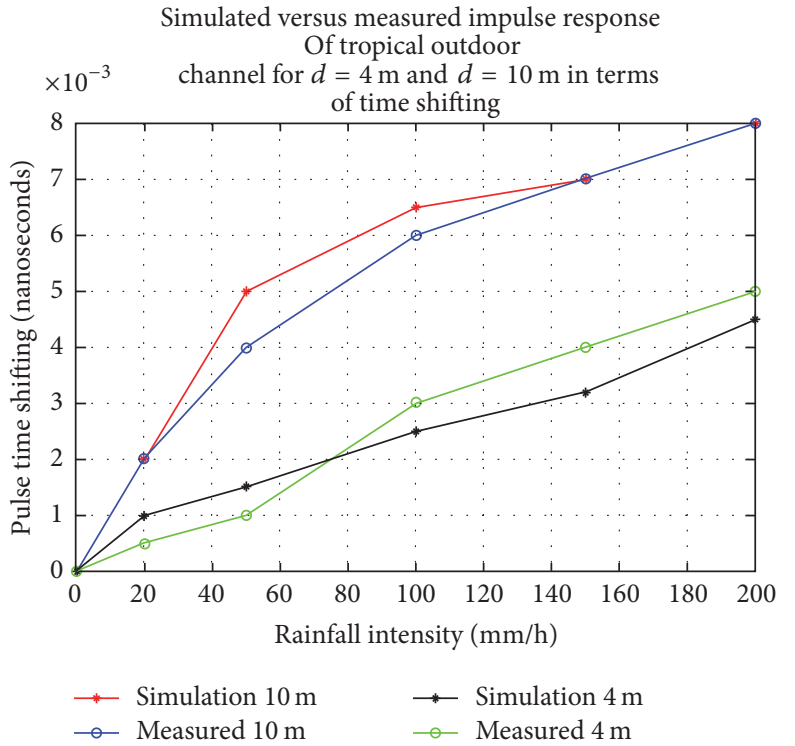

FIGURE 16: Simulated versus measured pulse time shifting of tropical outdoor channel at $d=4$ meters and $d=10$ meters.

performance of correlator-based UWB receiver without phase compensation.

However, for UWB systems with a minimum SNR and $10 \mathrm{~dB}$ fading margin of $5 \mathrm{~dB}, 10^{-6} \mathrm{BER}$ performance cannot be maintained because the phase compensation is not enough to overcome the performance degradation by dispersive antenna and rainfall intensity $120 \mathrm{~mm} / \mathrm{h}$. Correlator-based receiver with phase compensation can only achieve BER performance $10^{-4}$. In other words, the correlator-based UWB receiver does not meet the performance specifications that have been required. 


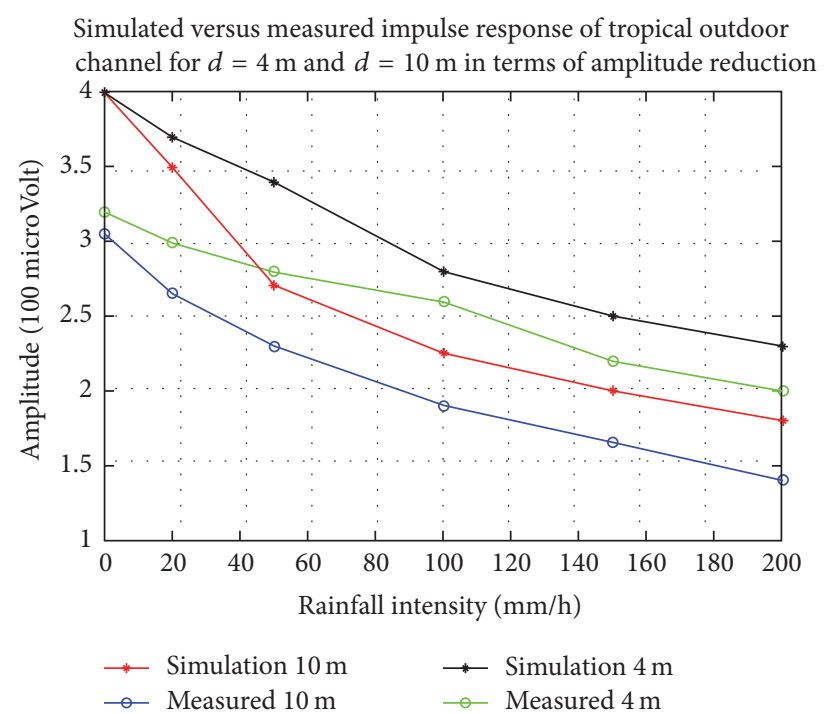

FIGURE 17: Simulated versus measured amplitude of tropical outdoor channel at $d=4$ meters and $d=10$ meters.

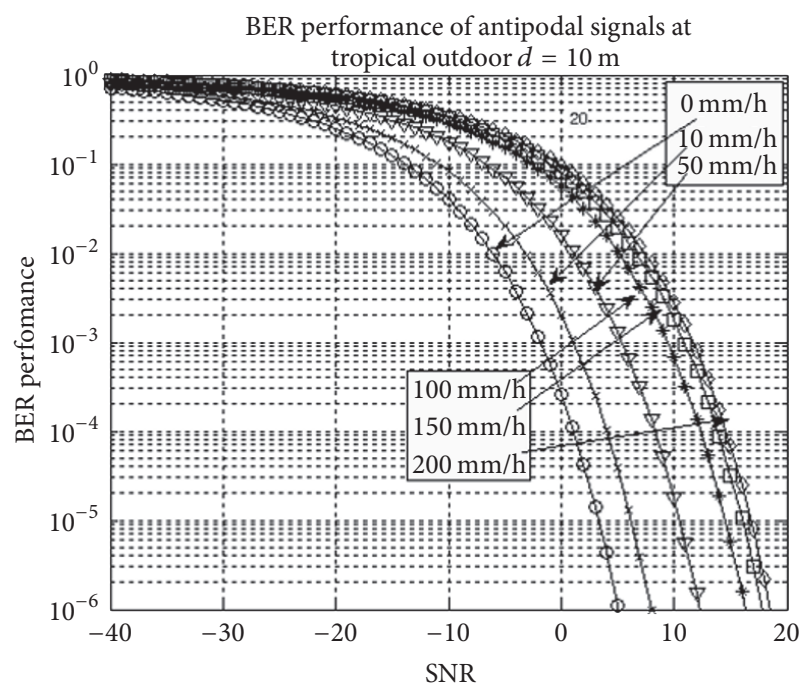

FIGURE 18: BER performance curves of matched filter-based receiver with ideal antenna.

\section{Conclusions and Future Research Direction}

Several important conclusions produced in this research are as follows:

(1) The dynamics of tropical outdoor channel versus time is strongly influenced by the atmosphere, especially the rainfall components. At a very high rainfall intensity $(200 \mathrm{~mm} / \mathrm{h})$, a tropical outdoor channel will have a large difference attenuation of low frequency components with the highest frequency component of the UWB band, that is, $9.5 \mathrm{~dB} / \mathrm{km}$. In addition, the frequency components of the UWB signal spectrum over the tropical outdoor channel will also shift in nonlinear phase by rain in its path components $0.3 \mathrm{Rad} / \mathrm{km}$ at the same rainfall. The results

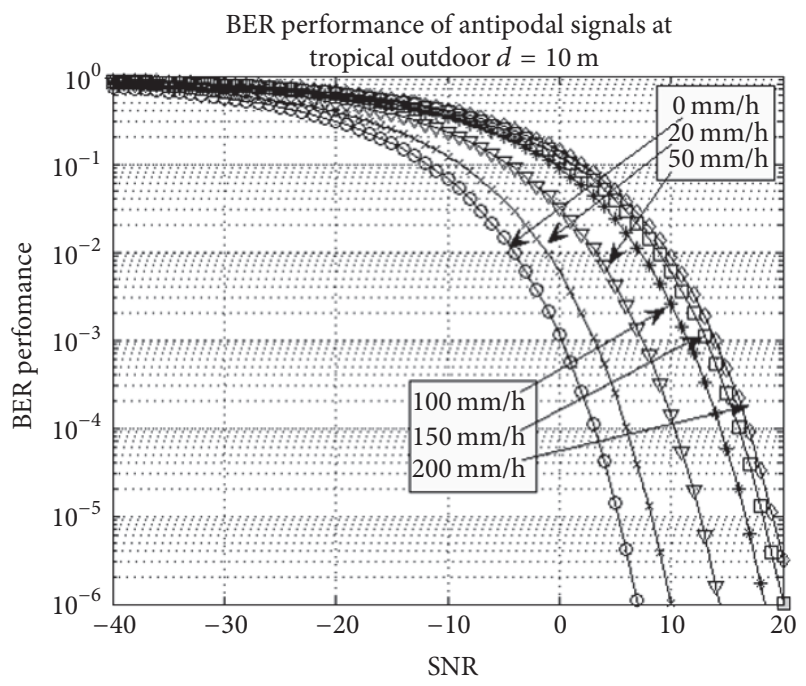

FIGURE 19: BER performance curves of correlator-based receiver with ideal antenna.

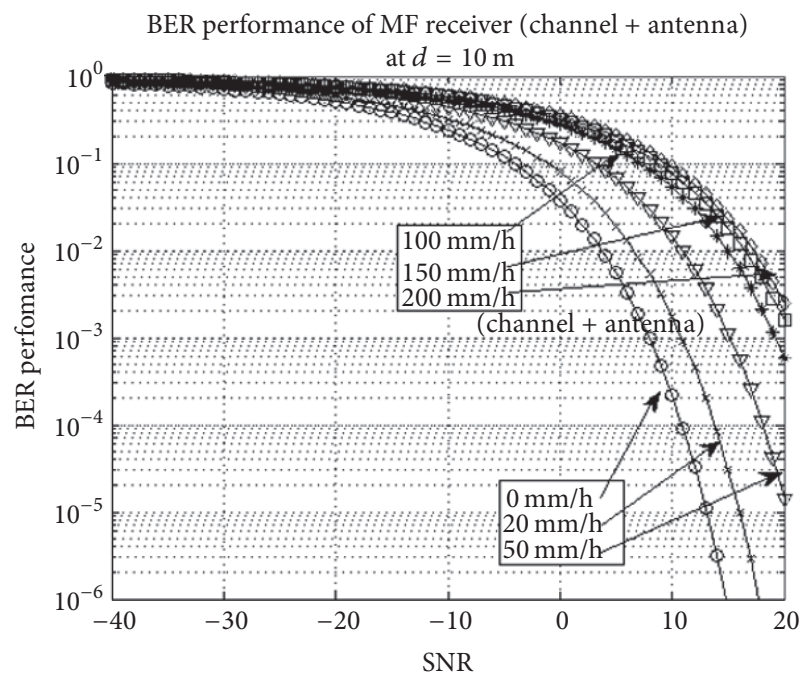

FIGURE 20: BER performance curves matched filter-based receiver with realistic UWB antenna.

of numerical simulations, the channel measurement, and mathematical models show the suitability of tropical UWB channel distortion causing a widening of pulse duration, the main axis, and a shift amplitude reduction.

(2) In no rain conditions, a BER performance at $10^{-6}$ can be achieved with the SNR $5 \mathrm{~dB}$, but at rainfall intensity at $200 \mathrm{~mm} / \mathrm{h}$, BER deteriorated to $10^{-2}$ for matched filter-based and for correlator-based falls to $5 \times 10^{-2}$. Rainfall intensity of $200 \mathrm{~mm} / \mathrm{h}$ can cause $15 \mathrm{~dB}$ loss of UWB signal quality for matched filter and $19 \mathrm{~dB}$ for correlator-based receiver. So in this case, the optimal system based on matched filter has a 3-5 dB better performance than correlator-based for tropical areas. The rainfall intensity has also a direct impact on the 


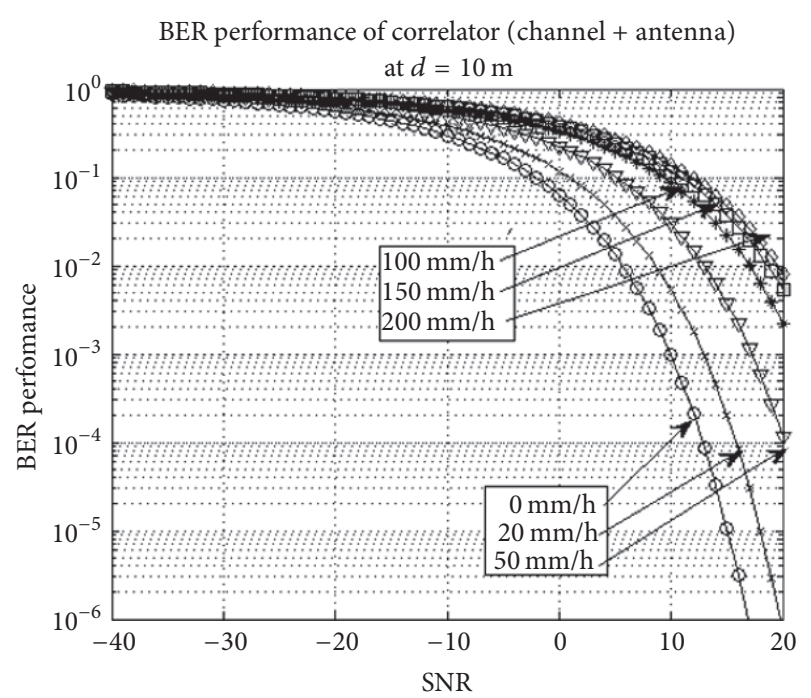

FIGURE 21: BER performance curves correlator-based receiver with realistic UWB antenna.

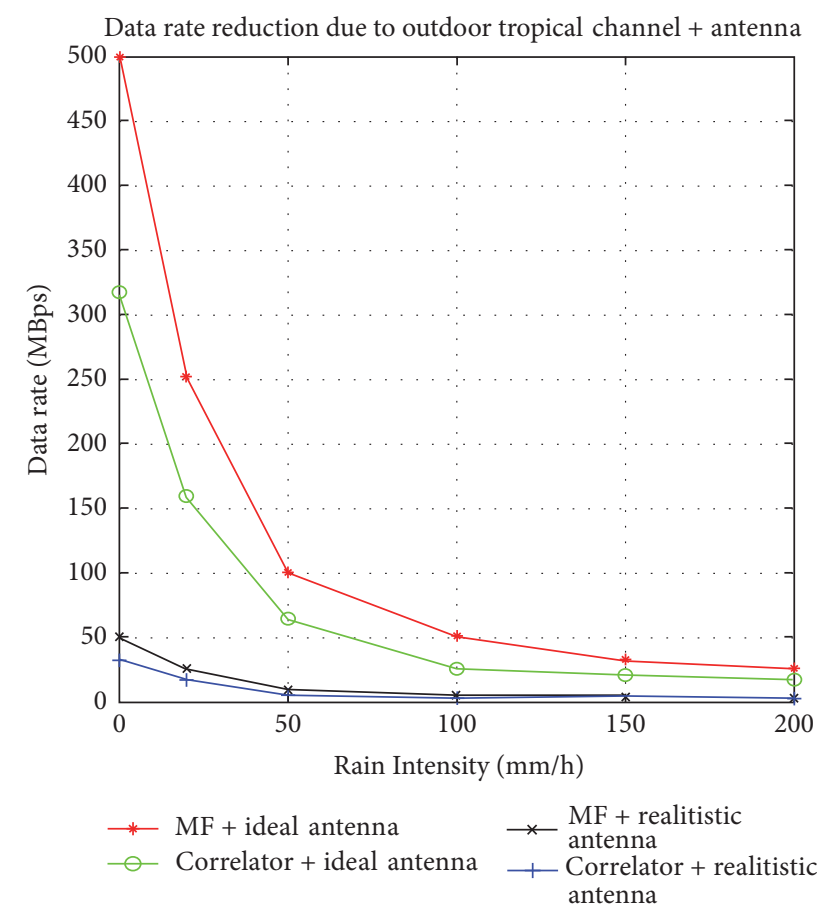

Figure 22: Bitrate reduction of UWB-based $5 \mathrm{G}$ system due to tropical outdoor channel and the antenna effects.

bitrate reduction on UWB communication systems. In an ideal scenario with ideal antennas, at rainfall intensity $200 \mathrm{~mm} / \mathrm{h}$, bitrate of UWB communication systems, will go down from $500 \mathrm{Mbps}$ (no rain) to 25 Mbps for the matched filter and 15 Mbps for the correlator-based receivers. Meanwhile, in a realistic antenna scenario, at rainfall intensity $200 \mathrm{~mm} / \mathrm{h}$, the bitrate of UWB communication systems declined from $50 \mathrm{Mbps}$ (no rain) to $2 \mathrm{Mbps}$ for matched filter and $1 \mathrm{Mbps}$ for the correlator-based receiver.
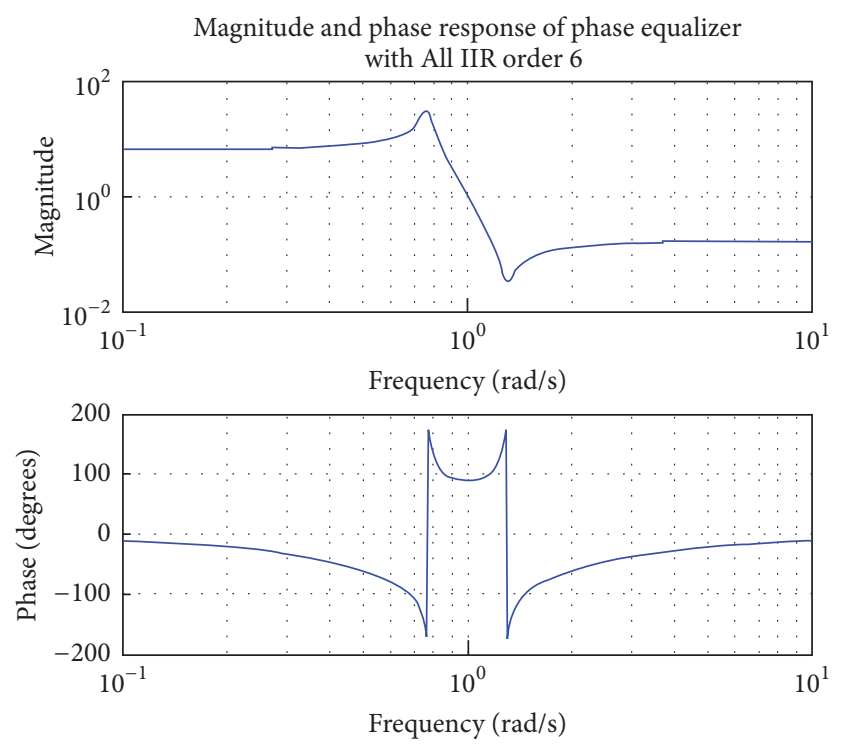

FIGURE 23: The magnitude and phase responses of proposed allpass IIR order 6 .

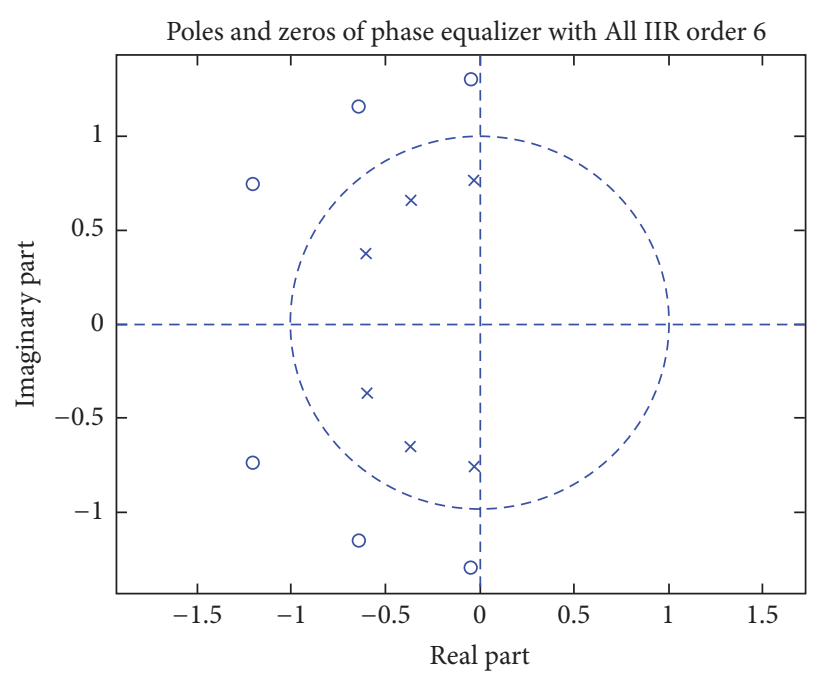

FIgURE 24: Poles and zeros structure of proposed Allpass IIR order 6.

(3) It can be known from the numerical simulation and measurement results of $1-13 \mathrm{GHz}$ tropical outdoor UWB channel that the pulse distortion is caused by the nonlinearity of phase responses and the magnitude response ruggedness of antenna and outdoor tropical channel. A proposed adaptive nonlinear phase equalizer with allpass IIR order 6 or more cascaded with a low-order FIR structure $(>6)$ can be used to compensate an accumulation of distortion by the transmission channel and the antenna.

Simulation results show that the $500 \mathrm{MBps}$ UWBbased 5G system performance using matched filterbased receiver at $10^{-6}$ BER can still be maintained for the $R_{0.01}$ rainfall intensity or availability of $99.99 \%$. In 


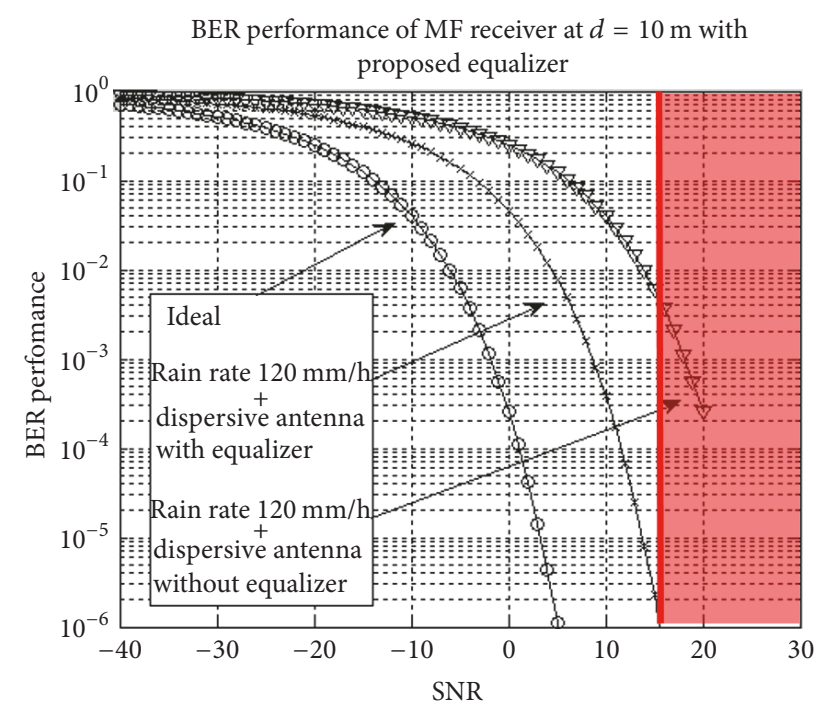

FIGURE 25: BER performance of the matched filter-based receiver with phase compensation.

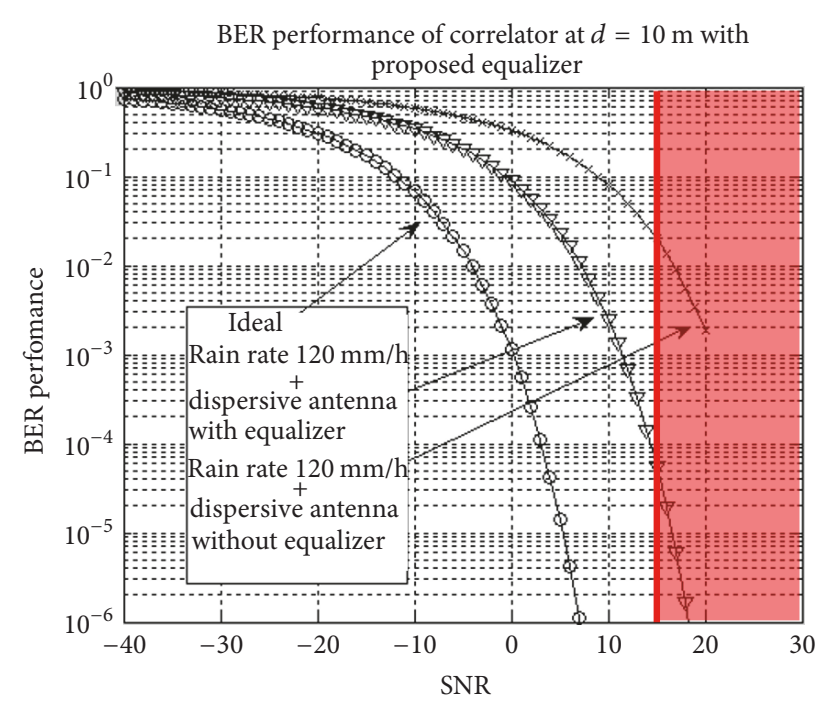

FIGURE 26: BER performance of the correlator-based receiver with phase compensation.

addition, the proposed nonlinear phase equalizer is capable of providing improved UWB signal by $9 \mathrm{~dB}$ at $120 \mathrm{~mm}$ rainfall/h compared with the UWB system without phase compensator. These results make us confident of bringing our future research to increase the target bit rate by exploiting the massive MIMO transceivers and using higher order modulation.

In the near future, we will perform field test of our $1-13 \mathrm{GHz} 10 \times 10$ MIMO based on PXI-based National Instruments SDR for UWB-based 5G system application at tropical environment. Our field test activities will be reported at the end of the year 2017, that is, during rainy season in Indonesia.

\section{Conflicts of Interest}

The author declares that he has no conflicts of interest.

\section{References}

[1] B. Van Lievenoogen, "Investigation of the suaitability of ultrawideband in military scenarios," Master Thesis Report, Wireless and Mobile Communciations Group, Delft University of Technology, 2008.

[2] N. A. Alsindi, B. Alavi, and K. Pahlavan, "Measurement and modeling of ultrawideband TOA-based ranging in indoor multipath environments," IEEE Transactions on Vehicular Technology, vol. 58, no. 3, pp. 1046-1058, 2009.

[3] J. A. N. Noronha, Ultrawideband channel sounding studies in outdoor and outdoor-indoor environments [thesis], Virginia Polytechnic Institute and State University, Blacksburg, Va, USA, 2004.

[4] L. Rubio, J. Reig, H. Fernández, and V. M. Rodrigo-Peñarrocha, "Experimental UWB propagation channel path loss and timedispersion characterization in a laboratory environment," International Journal of Antennas and Propagation, vol. 2013, Article ID 350167, 7 pages, 2013.

[5] C. J. Gibbins, "Propagation of very short pulses through the absorptive and dispersive atmosphere," IEE Proceedings $H$ : Microwaves, Antennas and Propagation, vol. 137, no. 5, pp. 304310, 1990.

[6] A. Maitra, M. Dan, A. K. Sen, K. Bhattacharyya, and C. K. Sarkar, "Propagation of very short pulses at millimeter wavelengths through rain filled medium," International Journal of Infrared and Millimeter Waves, vol. 14, no. 3, pp. 703-713, 1993.

[7] M. Z. Win, F. Ramirez-Mireles, R. A. Scholtz, and M. A. Barnes, "Ultra-wide bandwidth (UWB) signal propagation for outdoor wireless communications," in Proceedings of the 47th IEEE Vehicular Technology Conference, vol. 1, pp. 251-255, May 1997.

[8] B. Scheers, Ultra-wideband ground penetrating radar, with application to the detection of anti personnel landmines [Ph.D. dissertation], Laboratoire D'Hyperfrequences, Universite Catholique De Louvain, Ottignies-Louvain-la-Neuve, Belgium, 2001.

[9] M. Ghavami, L. B. Michael, and R. dan Kohno, Ultra Wideband Signals and Systems in Communication Engineering, John Wiley \& Sons, New York, NY, USA, 2nd edition, 2007.

[10] H. C. V. D. Hulst, Light Scattering by Small Particles, John Wiley \& Sons, New York, NY, USA, 1957.

[11] L. J. Ippolito, A Summary of Propagation Impairments on 10-100 GHz Satelite Links with Techniques, Propagation Effects Handbook for Satellite Systems Design, NASA Reference Publication 1082(04), NASA, 1989.

[12] H. J. Liebe, G. A. Hufford, and T. Manabe, "A model for the complex permittivity of water at frequencies below $1 \mathrm{THz}$," International Journal of Infrared and Millimeter Waves, vol. 12, no. 7, pp. 659-675, 1991.

[13] K. Teplee, "BER performance of UWB communications with matched filter and correlation receivers," in Proceeding of the ECTI International Conference, 2007.

[14] Z. N. Chen, X. H. Wu, H. F. Li, N. Yang, and M. Y. W. Chia, "Considerations for source pulses and antennas in UWB radio systems," IEEE Transactions on Antennas and Propagation, vol. 52, no. 7, pp. 1739-1748, 2004.

[15] J. S. Abel, "Robust design of very high-order allpass dispersion filters," in Proceedings of the 9th International Conference on 
Digital Audio Effects (DAFx '06), Montreal, Canada, September 2006.

[16] J. Suryana, S. Utoro, K. Tanaka, K. Igarashi, and M. Iida, “Two years characterization of concurrent $\mathrm{Ku}$-band rain attenuation and tropospheric scintillation in Bandung, Indonesia using JCSAT3," in Proceedings of the 5th International Conference on Information, Communications and Signal Processing (ICICS '05), pp. 1585-1589, December 2005.

[17] S. Slicul, Ultrawideband antena characterization and measurements [Ph.D. dissertation], Electrical Engineering, Virginia Tech, 2004.

[18] J. Suryana, A. B. Suksmono, Sugihartono, and A. Kurniawan, "Numerical and experimental investigations of a tropical outdoor UWB channel characteristics for short pulse transmission," in Proceedings of the PIERS, Tokyo, Japan, August 2006. 




\section{Enfincering}
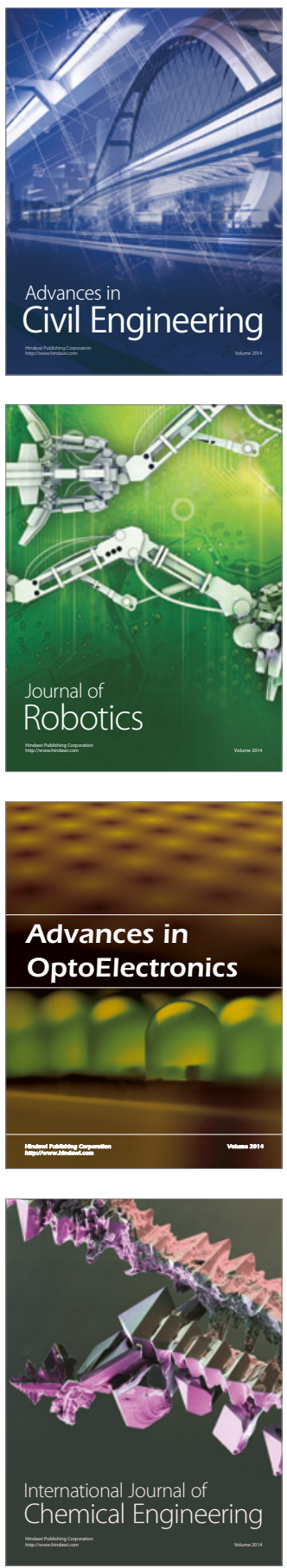



The Scientific World Journal



\section{Hindawi}

Submit your manuscripts at

https://www.hindawi.com
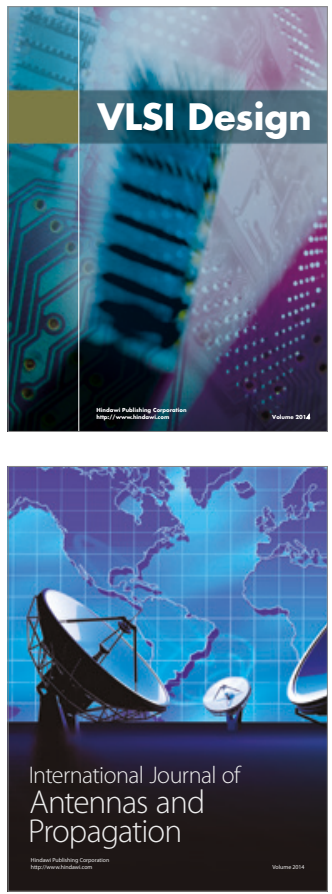

\section{Rotating}

Machinery
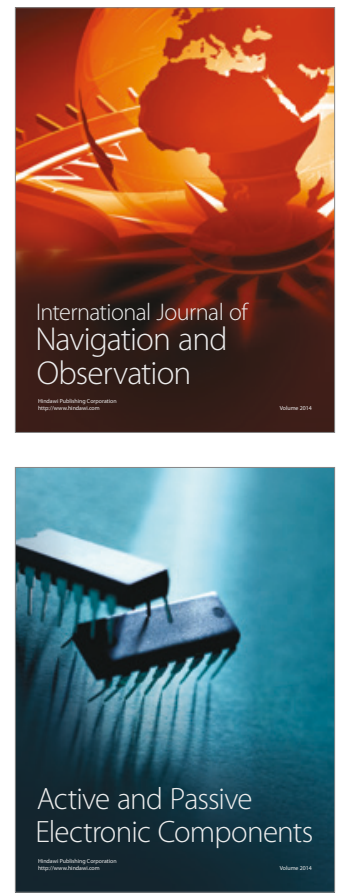
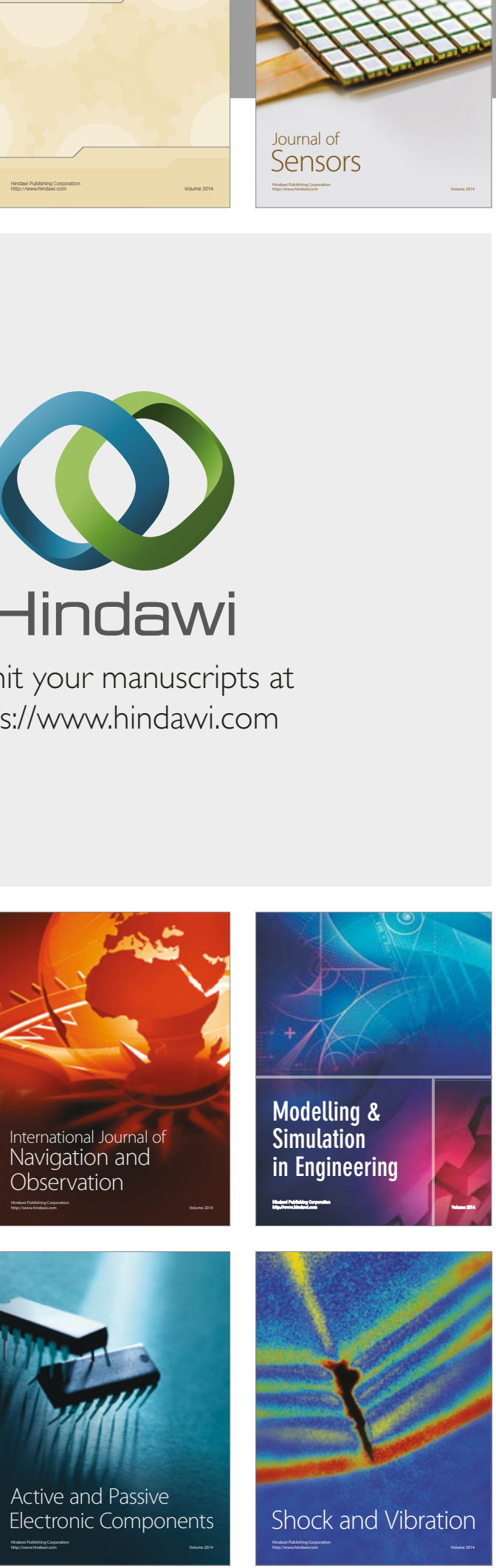
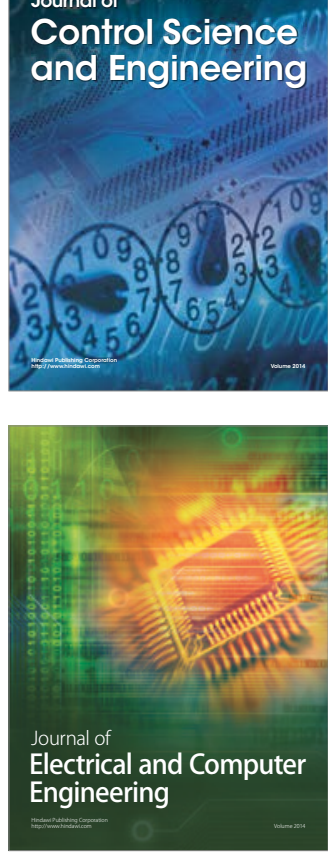

Distributed

Journal of

Control Science

and Engineering
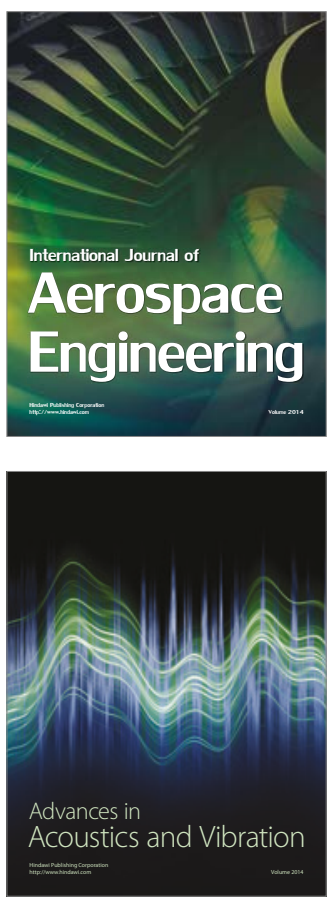

Sensor Networks 